\title{
Characterization of the Evolution of Noble Metal Particles in a Commercial Three-Way Catalyst: Correlation between Real and Simulated Ageing
}

\author{
Mattia Giuliano ${ }^{1,2, *}$, Maria Carmen Valsania ${ }^{1}$, Pierfrancesco Ticali ${ }^{1}$, Enrico Sartoretti ${ }^{3} \mathbb{D}$, Sara Morandi $^{1} \mathbb{D}$, \\ Samir Bensaid $^{3}$, Gabriele Ricchiardi ${ }^{1}$ (D) and Mauro Sgroi ${ }^{2}$ (D) \\ 1 Department of Chemistry, NIS and INSTM Reference Centre, University of Torino, Via G. Quarello 15, \\ I-10135 and Via P. Giuria 7, I-10125 Torino, Italy; mariacarmen.valsania@unito.it (M.C.V.); \\ pierfrancesco.ticali@unito.it (P.T.); sara.morandi@unito.it (S.M.); gabriele.ricchiardi@unito.it (G.R.) \\ 2 Batteries \& Aftertreatment, E/E \& Multifunctional Materials, Materials Engineering Methods \& Tools, \\ Fiat Research Center, Strada Torino 50, 10043 Orbassano, Italy; mauro.sgroi@crf.it \\ 3 Department of Applied Science and Technology, Politecnico di Torino, Corso Duca degli Abruzzi 24, \\ 10129 Turin, Italy; enrico.sartoretti@polito.it (E.S.); samir.bensaid@polito.it (S.B.) \\ * Correspondence: mattia.giuliano@crf.it; Tel.: +39-011-908-3605
}

Citation: Giuliano, M.; Valsania, M.C.; Ticali, P.; Sartoretti, E.; Morandi, S.; Bensaid, S.; Ricchiardi, G.; Sgroi, M. Characterization of the Evolution of Noble Metal Particles in a Commercial Three-Way Catalyst: Correlation between Real and Simulated Ageing. Catalysts 2021, 11, 247. https://doi.org/10.3390/ catal11020247

Academic Editors: Maria Casapu and Dmitry E. Doronkin

Received: 7 January 2021

Accepted: 9 February 2021

Published: 12 February 2021

Publisher's Note: MDPI stays neutral with regard to jurisdictional claims in published maps and institutional affiliations.

Copyright: (c) 2021 by the authors. Licensee MDPI, Basel, Switzerland. This article is an open access article distributed under the terms and conditions of the Creative Commons Attribution (CC BY) license (https:// creativecommons.org/licenses/by/ $4.0 /)$.

\begin{abstract}
Ageing of automotive catalysts is associated to a loss of their functionality and ultimately to a waste of precious resources. For this reason, understanding catalyst ageing phenomena is necessary for the design of long lasting efficient catalysts. The present work has the purpose of studying in depth all the phenomena that occur during ageing, in terms of morphological modification and deactivation of the active materials: precious metal particles and oxidic support. The topic was deeply investigated using specific methodologies (FT-IR, CO chemisorption, FE-SEM) in order to understand the behavior of metals and support, in terms of their surface properties, morphology and dispersion in the washcoat material. A series of commercial catalysts, aged in different conditions, have been analyzed, in order to find correlations between real and simulated ageing conditions. The characterization highlights a series of phenomena linked to the deactivation of the catalysts. $\mathrm{Pd}$ nanoparticles undergo a rapid agglomeration, exhibiting a quick loss of dispersion and of active sites with an increase of particles size. The evolution of the support allows highlighting also the contribution of chemical ageing effects. These results were also correlated with performance tests executed on synthetic gas bench, underlining a good correspondence between vehicle and laboratory aged samples and the contribution of chemical poisoning to vehicle aged ones. The collected data are crucial for the development of accelerated laboratory ageing protocols, which are instrumental for the development and testing of long lasting abatement systems.
\end{abstract}

Keywords: washcoat; sintering; ageing; chemisorption; FT-IR

\section{Introduction}

Automotive catalysts usually work in extremely harsh conditions: at high temperature due to the exothermic reactions involved, with local peaks above $1000-1100{ }^{\circ} \mathrm{C}$, and in a very variable chemical environment. This is particularly true in gasoline-fueled vehicles, where the main system adopted to reduce engine emissions is represented by the three-way catalyst (TWC) [1,2]. This catalytic system is able to perform simultaneous oxidation of $\mathrm{CO}$ and unburned hydrocarbons $(\mathrm{HC})$ and reduction of nitrogen oxides $\left(\mathrm{NO}_{\mathrm{x}}\right)$, thanks to a well-controlled air-to-fuel ratio that keeps the gaseous stream around stoichiometry [3]. These conditions promote a series of phenomena called thermal ageing which are the primary sources of catalyst deactivation [4-6]. The high temperature induces a series of physical processes that lead to a modification of the washcoat structure, generically defined as sintering. These phenomenon lead to a loss of active surface via structural modification of the porous support, with a decrease of surface area of the carrier $[7,8]$. Highly dispersed 
noble metals particles undergo similar processes. Sintering occurs either via dissolution of small particles and accretion of large ones, or via coalescence of particles trough migration of crystallites. Both processes are facilitated by surface solvation of mobile ionic species. The final result is the formation of larger agglomerates, with a reduction of the active surface exposed to reactants [9]. Platinum Group Metals (PGM) particles can be affected by the sintering process of the support, suffering encapsulation of due to the collapse of the porous structure [10]. The metal sintering obviously depends on the nature of the metal, on the temperature and, to a lesser extent, on the reaction atmosphere [11,12]. The overall result is a decay of the catalytic performance upon ageing. The increasingly stringent limits posed by emission regulations concern not only the initial performance of the catalysts, but also their durability, challenging automotive manufacturers to design and test emission control systems whose efficacy spans the entire working life of the vehicle [13]. In this context, laboratory simulation of ageing effects is crucial to the design of catalytic systems $[14,15]$. Beside fuel combustion products and by-products, the catalysts are in contact with numerous chemical species, which cause further deactivation by "chemical ageing". This is linked to the deposition on the catalyst of foreign elements carried by the exhaust gases, generated by the combustion of oil and fuel additives ( $\mathrm{Zn}, \mathrm{P}, \mathrm{Ca}, \mathrm{S}$ ), which can react with the oxides and noble metals present. These reactions can lead to the formation of less active compounds, and then decrease the catalyst performance, or to the deposition of an inactive surface layer, which makes it impossible for the gaseous reactants to access the pores of the washcoat [16]. Testing of catalyst ageing is crucial for catalytic systems design. However, direct on-vehicle testing is critical, because it is very time and labor consuming. Therefore, testing protocols executed on stationary engine bench facilities have been developed. However, their results do not entirely reproduce the real ageing happening upon the real vehicle usage. For these reasons, accelerated laboratory ageing methods are becoming more and more attractive [15-17]. The development of laboratory ageing protocols for automotive catalysts, able to overcome the limitations of accelerated engine bench tests, ensuring a better correlation with real on-vehicle ageing requires a deep knowledge of catalyst behavior in working condition during its entire useful life. Numerous studies have investigated this correlation on ad-hoc prepared catalysts [18-21], with the limitation that no on-vehicle data can be collected for these systems.

The present work has the purpose of comparing vehicle and laboratory aged catalysts in terms of morphological and chemical modifications and functional deactivation. In particular, the investigation is focused on the central element for the catalyst functionality: the evolution of noble metal particles. Deactivation was thoroughly investigated using specific methodologies in order to understand the evolution of PGM, and its correlation with ageing conditions. To this purpose, a series of recent commercial catalyst samples from a leading manufacturer were aged in different conditions and analyzed using different techniques in order to understand the evolution of the noble metal particles. In situ FTIR probe molecule experiments and pulse calorimetry experiments, both using $\mathrm{CO}$ as probe molecule, were used to probe the active sites of the catalyst. Transmission Electron Microscopy (TEM), with associated Energy Dispersive X-Ray Analysis (EDX) provided a complementary structural insight into particles evolution. Finally, functional catalytic testing of the different aged samples provided the framework for the interpretation of the results, suggesting strategies for developing better simulated ageing methods.

\section{Results}

\subsection{FTIR Surface Characterization of the Metal Particles and of the Support}

We consider now the characterization of noble metal surface sites and the evaluation of sintering phenomena by means of in situ FTIR experiments with $\mathrm{CO}$ as a probe molecule. Noble metals are present in the samples in very low concentration (1-2 wt.\%), making it very difficult to follow specifically their evolution during ageing. Carbon monoxide is a very versatile molecule, widely used to probe both the acid/base properties of the surfaces of oxides and adsorption on metallic phases Due to the high affinity of noble metals for 
$\mathrm{CO}$, adsorption on metal particles is detectable at room temperature, while adsorption on the support oxides requires low temperatures [21-23]. In a first experiment, $\mathrm{CO}$ adsorption at Room Temperature (RT) was performed on the washcoat powder extracted from a fresh catalyst sample (see the Materials and Methods Section below). The sample was outgassed in vacuum at $400{ }^{\circ} \mathrm{C}$ for $30 \mathrm{~min}$. The absorbance spectra recorded upon $\mathrm{CO}$ dosage (not shown) did not show any trace of peaks associated to the interaction between the probe gas molecule and noble metal particles, irrespective of the partial pressure of $\mathrm{CO}$ on the sample. This lack of reactivity of the fresh catalyst was interpreted as originating from the low severity of the surface outgassing protocol, which did not clean the surface from foreign adsorbed species. In order to eliminate these species, an oxidationreduction pretreatment was adopted. The treatment with $\mathrm{O}_{2}$ eliminates organic adsorbed contaminants by oxidation, and the successive reduction with $\mathrm{H}_{2}$ reactivate the metal surface, reducing the eventually oxidized metal sites. Due to the better results obtained with the oxidation-reduction pretreatment, this was applied also to all the other samples, which were exposed to the ageing environment $(25,000 \mathrm{~km}, 60,000 \mathrm{~km}$, Lab-aged). In Figure 1 the $\mathrm{CO}$ adsorption measurements performed at $\mathrm{RT}$ after the oxidation-reduction pretreatment are presented. For the fresh samples (Figure 1a), IR spectra show two peaks, with intensity that increases with the increase of pressure of $\mathrm{CO}$ dosed to sample. The first one is around $2100-2000 \mathrm{~cm}^{-1}$, typical of the stretching vibration of CO adsorbed in the on-top position on $\mathrm{Pd}^{0}$ site, and the second peak is around $2000-1700 \mathrm{~cm}^{-1}$, typical of CO stretching in bridge-bonded configuration between two $\mathrm{Pd}^{0}$ sites $[24,25]$. Increasing the $\mathrm{CO}$ pressure, the peaks gain in intensity and show a progressive shift to higher wave numbers, due to the increase of the $\mathrm{CO}$ coverage, which enhance the dipole coupling [26]. Upon outgassing, the $\mathrm{CO}$ coverage decreases and this causes a red-shift towards the original position. The highest occupied molecular orbital (HOMO) of $\mathrm{CO}$ has a slightly anti-bonding character. Therefore, when transition metal centers are involved, thanks to the $\sigma$-donation and $\pi$-backdonation effects, involving the HOMO and LUMO of CO and the uppermost $d$ states of the metallic phase, the appearance of absorption bands towards higher or lower frequency with respect to the gas phase $\left(2143 \mathrm{~cm}^{-1}\right)$ is expected [27]. The relative amount of on-top vs. bridged carbonyls can provide information on metal particles dispersion. However, the quantitative determination of the distribution of $\mathrm{CO}$ between on-top and bridge positions is very difficult and it was not possible on the collected data due to the overlap of the peaks. However, qualitative trends could be estimated. Pd tends to form bridged carbonyls when reduced, which are more stable than the linear ones [28]. A high contribution of the linearly bonded $\mathrm{CO}$ is an indication of a loss of dispersion of the $\mathrm{Pd}$, because the proximity of two Pd atoms is necessary to form bridges. In Figure 1b, the IR spectra recorded at increasing CO pressure on 25,000 km washcoat powder sample are presented. Comparing the spectra of the fresh sample with those of the $25,000 \mathrm{~km}$ one (sample aged on vehicle after 25,000 km operation, see Materials and Methods), the overall intensity of carbonyl bands is highly decreased, indicating a loss of Pd active sites. This effect can be linked to sintering phenomena occurred at high temperature, leading to a growth of noble metal particles and to a loss of surface of the porous support, that could lead to an encapsulation of nanoparticles, making them completely inaccessible to the probe molecule. These effects are enhanced on the sample aged on vehicle for 60,000 km (60 k) (Figure 1c), where the absorbance peaks associated to $\mathrm{CO}$ adsorption have completely disappeared. A previous work had shown a good correlation between the $60,000 \mathrm{~km}$ samples and that aged in the laboratory at $1100{ }^{\circ} \mathrm{C}$ for $7 \mathrm{~h}$ in hydrothermal condition (air-Lab-aged) [29]. In order to verify this, the latter was analyzed using the CO probe technique. Even in this case, the stretching peaks of adsorbed $\mathrm{CO}$ were not detectable (spectra not reported for sake of brevity). Given the non-detectability of CO on metal particles on the $60,000 \mathrm{~km}$ sample, the more aged samples $80,000 \mathrm{~km}$ and FUL (Full Useful Life, see below) were not studied with this technique. Finally, it is worth noticing that the $\mathrm{CO}$ adsorption experiments did not detect distinct peaks for $\mathrm{CO}$ adsorption on $\mathrm{Rh}$, which is present on the catalyst with 1:11 ratio with Pd, clearly too low for detection. We recognize that this is a limitation of our 
study, due to the great importance of $\mathrm{Rh}$ for $\mathrm{NO}_{\mathrm{x}}$ reduction functionality and to its peculiar deactivation mechanism [30], which should be addressed with more sensitive methods.

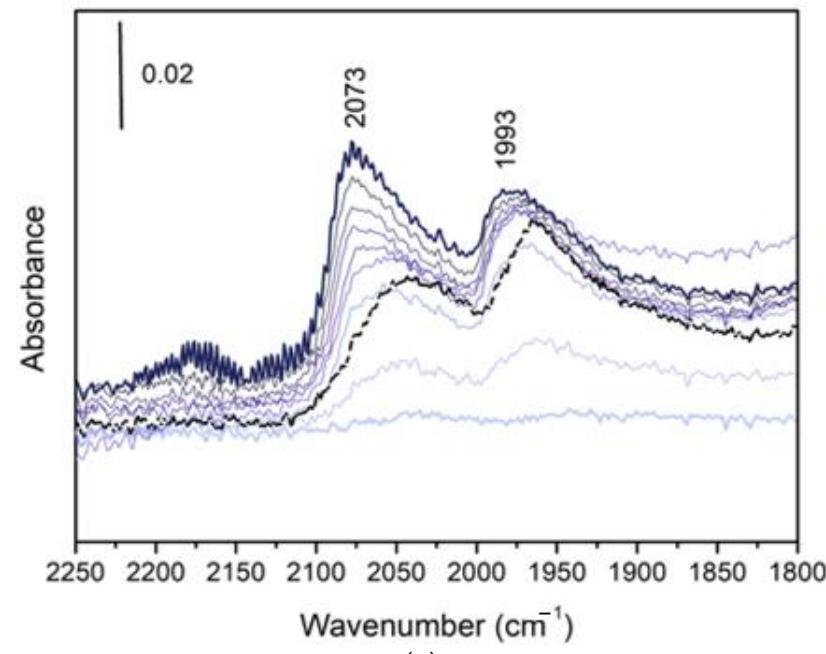

(a)

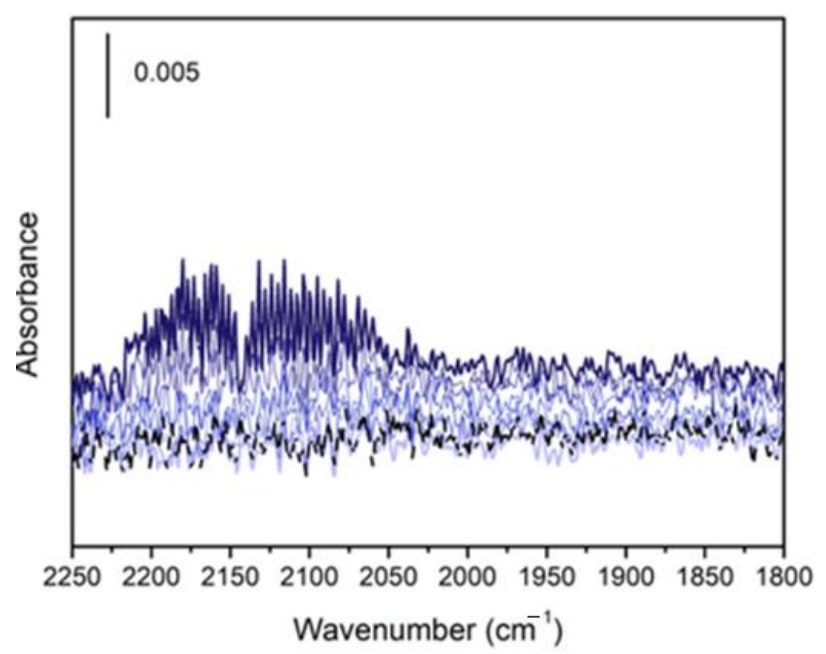

(c)

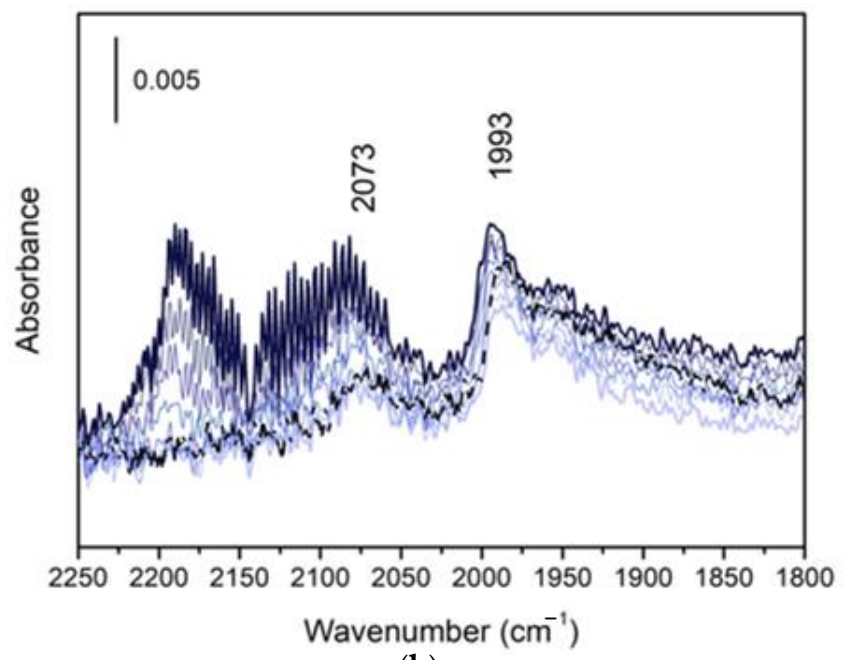

(b)

Figure 1. FT-IR spectra of CO adsorption at RT on fresh (a), 25,000 km (b) and 60,000 km (c) sample after $400{ }^{\circ} \mathrm{C}-0.5 \mathrm{~h}$ oxidation-reduction $\left(\mathrm{O}_{2}-\mathrm{H}_{2}\right)$ pretreatment at increasing pressure of $\mathrm{CO}$ up to $20 \mathrm{mbar}$ and after final outgassing (black dotted line).

Infrared analyses of the oxidic support with $\mathrm{CO}$ as a probe molecule require performing the adsorption at low temperature, due to the low binding energies involved. The support has an active role in the TWC and a complete characterization of these materials requires to understand whether the aging influences the support and in particular the oxygen storage capacity (OSC) of the material. This is the ability of the Ce-Zr mixed oxides support to adsorb/release oxygen during operation, storing it during a lean phase and releasing it during a rich one. OSC closely correlates with catalytic performances, because it leads to an increase of TWC efficiency by enlarging the air-to-fuel operating window [20]. Some samples were analyzed by $\mathrm{CO}$ adsorption at liquid nitrogen temperature (LNT). Figure 2 shows the spectra recorded on the fresh sample. They show an intense peak in the range 2200-2100 $\mathrm{cm}^{-1}$, whose intensity increases with CO pressure. The peak red-shifts from 2177 to $2155 \mathrm{~cm}^{-1}$ upon increasing CO coverage due to chemical effect, i.e., on increasing $\mathrm{CO}$ adsorbed on the surface, the $\sigma$-donation decreases. Consequently, electron density in the slightly antibonding $\mathrm{HOMO}$ orbital of $\mathrm{CO}$ increases and the $\mathrm{CO}$ stretching decreases. This peak is probably generated by the superposition of different contributions of $\mathrm{CO}$ 
adsorbed on $\mathrm{Ce}^{4+}$ and $\mathrm{Zr}^{4+}$ cations, since their stretching vibrations fall in this spectral region [22]. The convolution in one peak of these contributions, which should be separated in the pure oxides, can be associated to a compositionally homogeneous solid solution of the $\mathrm{Ce}$ and $\mathrm{Zr}$ oxides, with negligible contribution of segregated pure oxide phases. At lower wavenumbers bands related to on-top $\left(2086 \mathrm{~cm}^{-1}\right)$ and bridged $\left(1997\right.$ and $\left.1940 \mathrm{~cm}^{-1}\right)$ $\mathrm{Pd}^{0}$ carbonyls are present [25]. Differently from measurements performed at RT, at LNT the bridged carbonyl region shows complex features with the presence of two bands. Typically the IR spectra of $\mathrm{CO}$ adsorbed on reduced $\mathrm{Pd}$ are interpretable on the basis of what occurs on the most stable $\mathrm{Pd}$ faces, $\operatorname{Pd}(111)$ and $\operatorname{Pd}(100)[31,32]$. The peak at $1997 \mathrm{~cm}^{-1}$ is assigned to bridged carbonyls on Pd (100) faces, while the peak at $1940 \mathrm{~cm}^{-1}$ is related to bridged species on Pd (111) face. The band at $2086 \mathrm{~cm}^{-1}\left(2060 \mathrm{~cm}^{-1}\right.$ for very low CO coverage) is related to linear carbonyls on Pd (111) face. As observable at RT as well, the band of linear carbonyls is asymmetrical due to a shoulder on the low wavenumber side. This shoulder is reasonably due to the presence of defects such as edges, corners and kinks, on noble metal particle surface [33]. The stability of the carbonyl species during the outgassing (dashed lines in the figures) further helps to discriminate between $\mathrm{CO}$ bonded to $\mathrm{Pd}$ and to the support. Pd carbonyls are more stable than carbonyls of the support, since $\mathrm{Pd}^{0}$ bonds $\mathrm{CO}$ both by $\sigma$-donation and $\pi$-backdonation, while $\mathrm{Zr}$ and Ce cations bond $\mathrm{CO}$ exclusively via $\sigma$-donation and the related bands are completely removed by outgassing. These peaks display the same behavior observed at RT in aged samples, with a progressive disappearance of the signal due to sintering of noble metal particles (Figure 2).

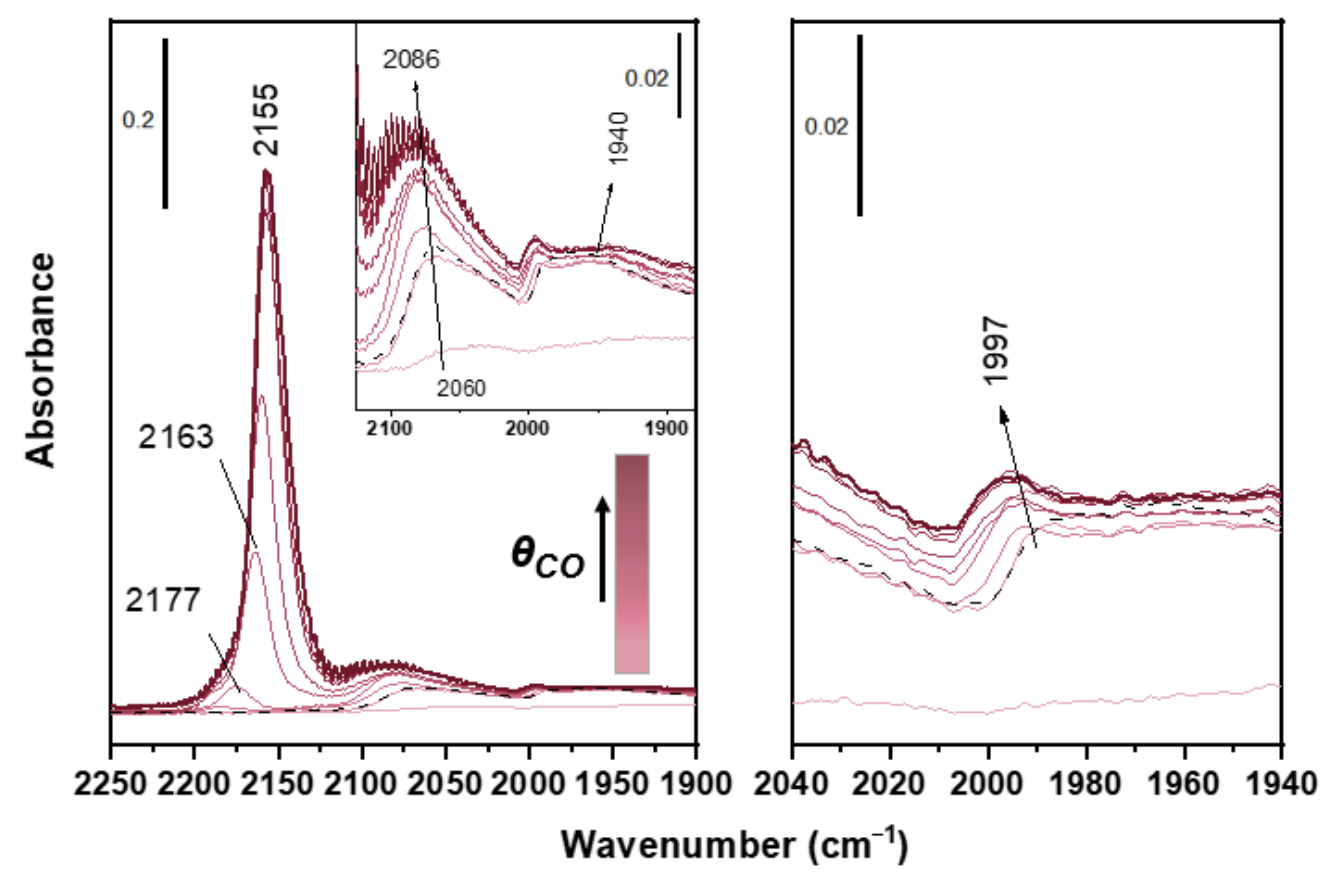

Figure 2. FT-IR spectra of $\mathrm{CO}$ adsorption at LNT on fresh sample after $400{ }^{\circ} \mathrm{C}-0.5 \mathrm{~h}$ oxidationreduction $\left(\mathrm{O}_{2}-\mathrm{H}_{2}\right)$ pretreatment at increasing pressure of $\mathrm{CO}$ up to $20 \mathrm{mbar}$ and after final outgassing at LNT (black dotted line).

The peaks associated to adsorption on the support material display a similar behavior: the intensity decreases upon ageing, due to the sintering of Ce-Zr oxides. This phenomenon causes not only a loss in intensity of the band but also a decrease in the chemical effect that causes a shift to higher frequencies at maximum coverage with respect to the fresh case. In particular, in the $60 \mathrm{k}$ sample (Figure 3a) a peak assigned to mono carbonyls on reduced $\mathrm{Ce}^{3+}$ species appears at $2116 \mathrm{~cm}^{-1}$ (see Figure 3 insets). The assignment of this band to the carbonyls on the support cation is corroborated by the low stability of the species, which are removed by outgassing (dashed line in Figure 3a). The appearance of this peak can 
be linked to the effects of ageing: the high temperature sintering of these components hindered the $\mathrm{Ce}^{4+}$ to $\mathrm{Ce}^{3+}$ redox reaction [34]. In addition, there may be a contribution of chemical ageing, with Ce reacting with the phosphate compound present in the engine out gas, coming from the combustion of engine oil additives, forming the very stable $\mathrm{CePO}_{4}$ phase [16-35]. These effects lead to immobilization of $\mathrm{Ce}$, hindering its participation to redox reactions necessary for oxygen storage. In this case, the peak can be due to both this effect and is consistent with the reduction of the OSC observed during the performance tests (see below). The laboratory-aged (Figure 3b) show a less intense peak associated to $\mathrm{Ce}^{3+}$, probably because in this case there is only the support sintering contribution. This sample has not been in contact with chemical species deriving from the combustion, so the chemical ageing effects in this case are not expected. As for the laboratory-aged sample, the contribution of $\mathrm{Pd}^{0}-\mathrm{CO}$ at $2096 \mathrm{~cm}^{-1}$ is quite evident. Moreover, it is worth to note that in the $60,000 \mathrm{~km}$ sample $\mathrm{Pd}^{0}$-CO might be associated to the shoulder of the peak at $2116 \mathrm{~cm}^{-1}$, the $\mathrm{Ce}^{3+}-\mathrm{CO}$ band, which is absent for the laboratory aged one.

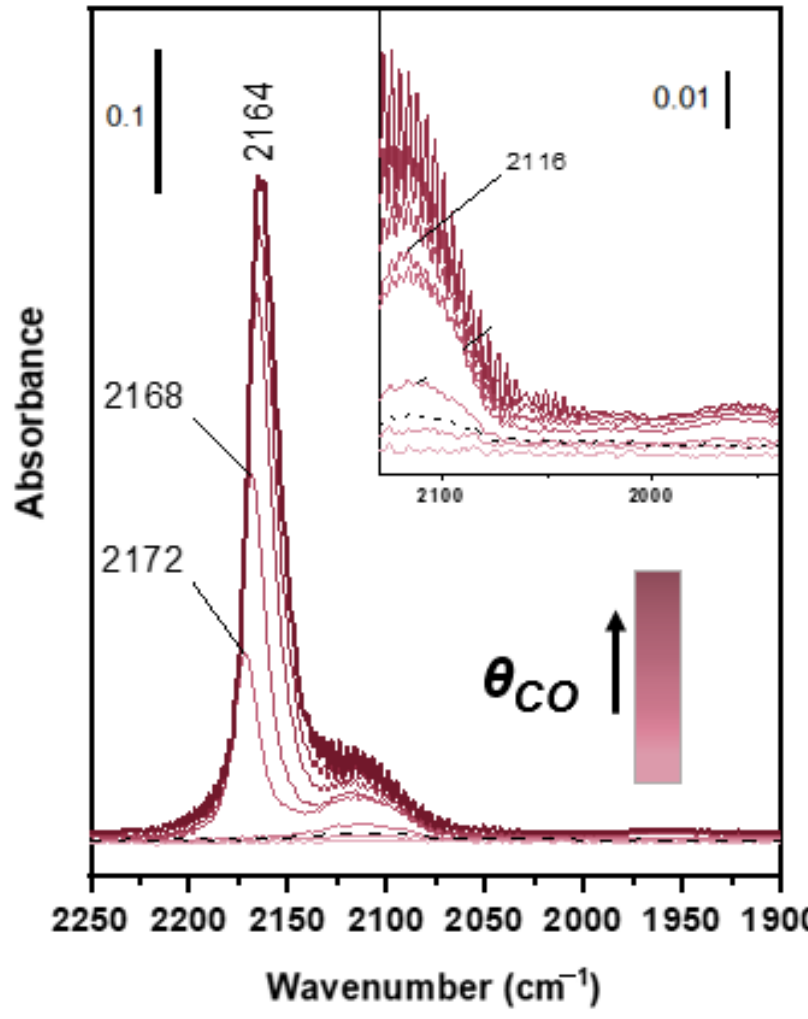

(a)

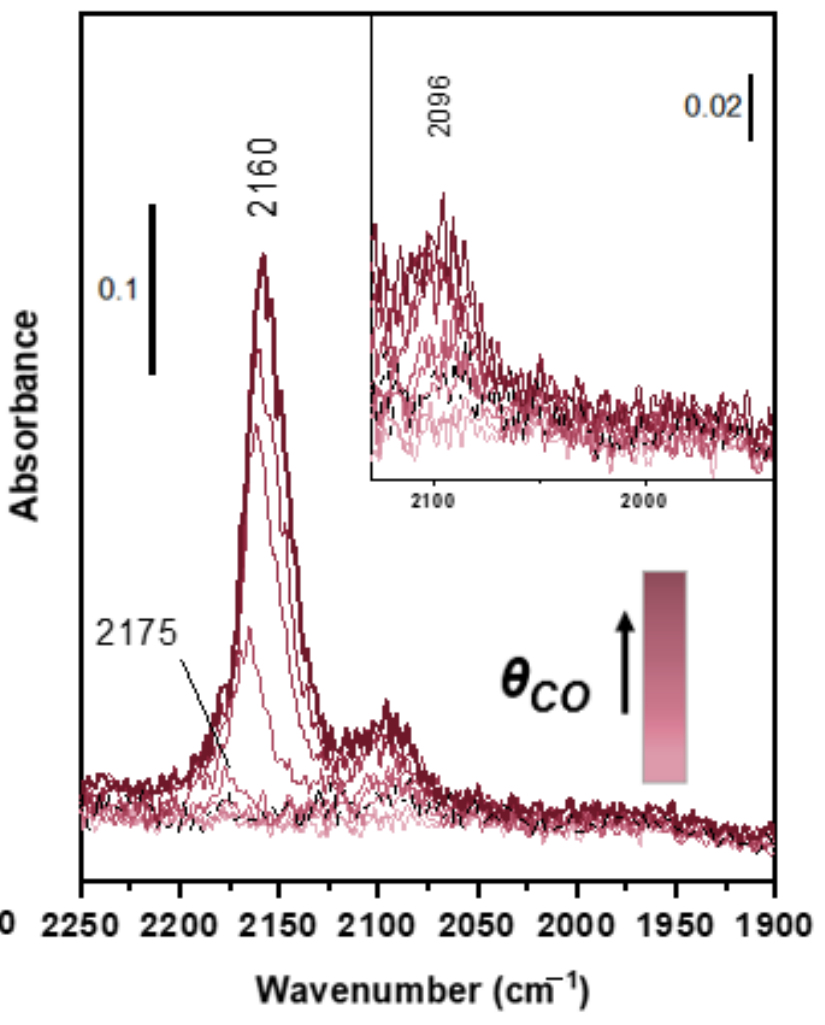

(b)

Figure 3. FT-IR measurement of CO adsorption at LNT on $60,000 \mathrm{~km}(\mathbf{a})$ and Lab-aged (b) after $400{ }^{\circ} \mathrm{C}-0.5 \mathrm{~h}$ oxidationreduction $\left(\mathrm{O}_{2}-\mathrm{H}_{2}\right)$ pretreatment at increasing pressure of $\mathrm{CO}$ up to 20 mbar and after final outgassing at LNT (black dotted line).

\subsection{Evaluation of Metal Particles Dispersion by CO Pulse Adsorption.}

The dispersion of the active metal particles has been studied with quantitative pulse $\mathrm{CO}$ chemisorption experiments, which allow to determine the adsorbed amounts and to model approximately the particle size [36]. The results of the CO-pulse experiment on the fresh sample are presented in Figure $4 \mathrm{a}, \mathrm{b}$, reporting the instantaneous and integrated TCD signal (proportional to the CO concentration at the reactor outlet). The first peaks are attenuated due to the chemisorption of $\mathrm{CO}$ on the surface of noble metal particles, while successive pulses gradually increase until saturation of the adsorption capacity of the metal, when the peaks reach a constant intensity. The $\mathrm{CO}$ uptake was calculated from the integral of the peaks (Figure $4 \mathrm{~b}$ ). The dispersion of Pd particles was calculated from 
the Pd:CO ratio. CO molecules can chemisorb on superficial Pd atoms in linear or bridged form and the coexistence of both the configurations was confirmed by IR spectroscopy. Since two IR peaks with similar intensities appearing after exposing the catalyst to CO, were ascribed to the two $\mathrm{CO}$ chemisorbed species, and considering their very similar extinction coefficient (accordingly with literature), a Pd:CO ratio of 1.5 can be suitably assumed for calculations [37]. Using this assumption, from the amount of chemisorbed CO it is possible to calculate the number of $\mathrm{Pd}$ sites involved in the reaction and evaluate the ratio between this value and the quantity of Pd present inside the sample, obtained from chemical analysis ( $1 \% \mathrm{Pd}$ in weight). The fresh sample exhibits a quite high palladium dispersion, around 51\%, expressed in terms of ratio between the moles of Pd involved in total CO uptake and total Pd moles in the sample. This result indicate that the noble metal is distributed on the support in very small particles, consistently with the absence of visible Pd particles in the SEM observations reported in the following. In fact, an average metal particle size of about $2 \mathrm{~nm}$ can be estimated from CO chemisorption.

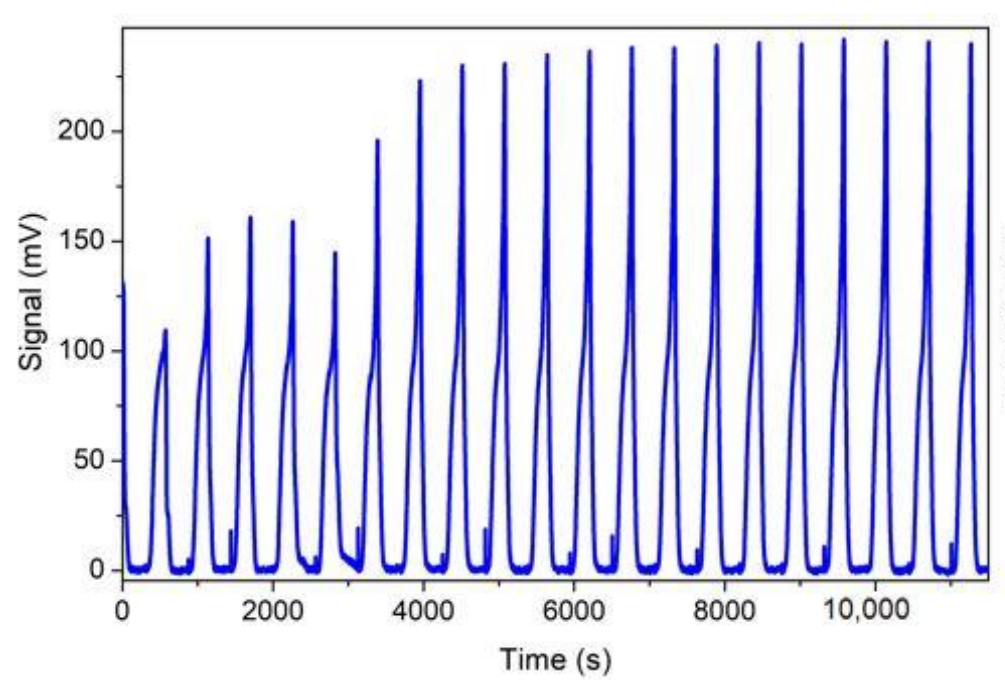

(a)

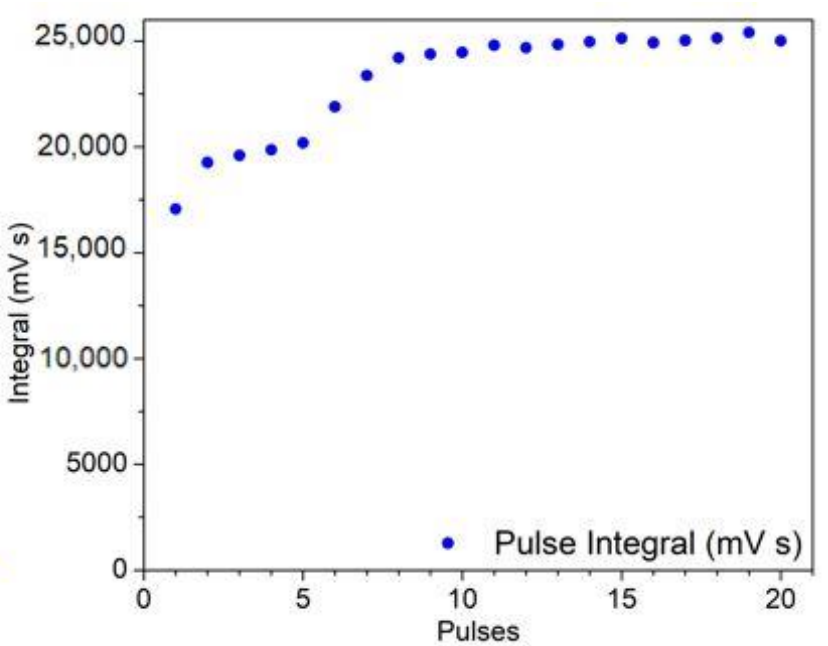

(b)

Figure 4. Fresh catalyst sample: (a) TCD signal $(\mathrm{mV})$ registered during successive sending of pulse of $800 \mu \mathrm{L}$ of $10 \% \mathrm{CO}$ in He carrier until saturation (b) Value of integral of the peaks registered.

For the $25,000 \mathrm{~km}$ sample, the situation changes significantly: the differences between the intensity of first peaks and peaks at saturation is much smaller, and the adsorbed amount is determined with low accuracy (please note the different scales of Figures 4 and 5).

The dispersion of Pd in this sample is decreased to $8 \%$, and for the $60,000 \mathrm{~km}$ catalyst, it is below $1 \%$, although in the latter cases the $\mathrm{CO}$ uptake is too low to obtain reliable values. These results point out that the metal particles are sensitive to sintering during the catalyst life cycle, as suggested by FT-IR measurement. The CO chemisorption test was performed also on laboratory-aged sample, in order to verify the correlation with $60,000 \mathrm{~km}$ one. Even in this case the data obtained do not allow to evaluate accurately the dispersion, which results below $1 \%$. Due to the results obtained on these samples, samples from catalysts with higher ageing $(80,000 \mathrm{~km}$ and FUL) were not analyzed.

\subsection{SEM Measurement of Metal Particles Dimensions.}

In a previous work [29], TEM images collected on fresh and FUL samples did not allow to observe the metal nanoparticles, due to the low contrast between noble metals and the substrate. The fresh sample washcoat displayed a coarse morphology, with porosity spanning a wide range of length scales. This porous structure was gradually 
lost with ageing, which showed a more compact washcoat [29]. In this work, we have collected high-resolution (HR) FE-SEM (Field Emission-Scanning Electron Microscopy) images of the different aged samples, highlighting the morphological effects of support and metal particles sintering. FE-SEM images of all the aged samples show the presence of bright small particles of uniform size, and a variable amount of large bright particles of different sizes, dispersed in the washcoat support. Figure 6 shows the case of the FUL sample. In order to identify these components, a series of EDX measurements, analyzing punctually these particles and recording a mapping on whole samples, was performed. The elements mapping highlights a high concentration of Pd in correspondence of lighter particles. Performing a single-point analysis on these bright zone, the presence of only $\mathrm{Pd}$, $\mathrm{O}$ and $\mathrm{C}$ is observed where the dimensions of particles are sufficiently large to shadow the contribution from the support. Therefore, it is possible to identify these components as $\mathrm{Pd} / \mathrm{PdO}$ particles, growth due to sintering of catalytic material. On the contrary, in the fresh sample, no particles were detected, but performing EDX analysis in different points, a constant Pd signal was recorded, originating from a uniform distribution of well dispersed nanoparticles. This is in agreement with CO chemisorption analysis.

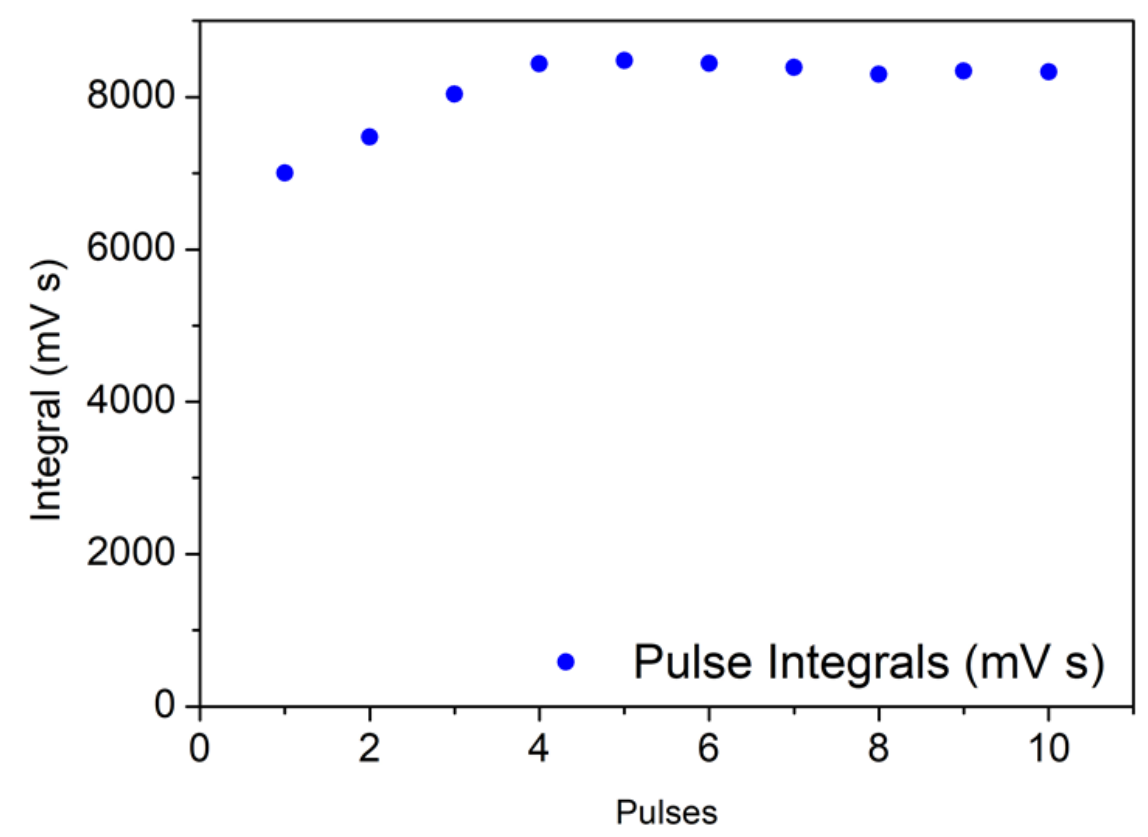

Figure 5. $25,000 \mathrm{~km}$ sample: integrals of TCD signal peaks registered during successive manual dosage of pulses of $800 \mu \mathrm{L}$ of $10 \% \mathrm{CO}$ in He carrier, until saturation.

A series of diameter measurements was taken, in order to quantify the particles size growth upon ageing.

Table 1 presents the minimum-maximum diameter values recorded on samples analyzed and the average value. The values presented are the results of measurement performed on 20 different Pd particles, detected in the same image. The diameter of noble metal particles increases with the ageing, overcoming $100 \mathrm{~nm}$ of average value from $60,000 \mathrm{~km}$ sample. This is in agreement with the results obtained by CO chemisorption followed by FT-IR and CO chemisorption measurements, which highlighted a high sintering of noble metal particles already in the $25,000 \mathrm{~km}$ sample. With a particle diameter above $100 \mathrm{~nm}$ the interaction of $\mathrm{CO}$ with the catalyst is limited, not enough to have to a complete deactivation of the material, but leading to results below the detection limits of the applied techniques. FUL sample is confirmed to be the worst case, with Pd agglomerates up to $0.8 \mu \mathrm{m}$ in diameter. 

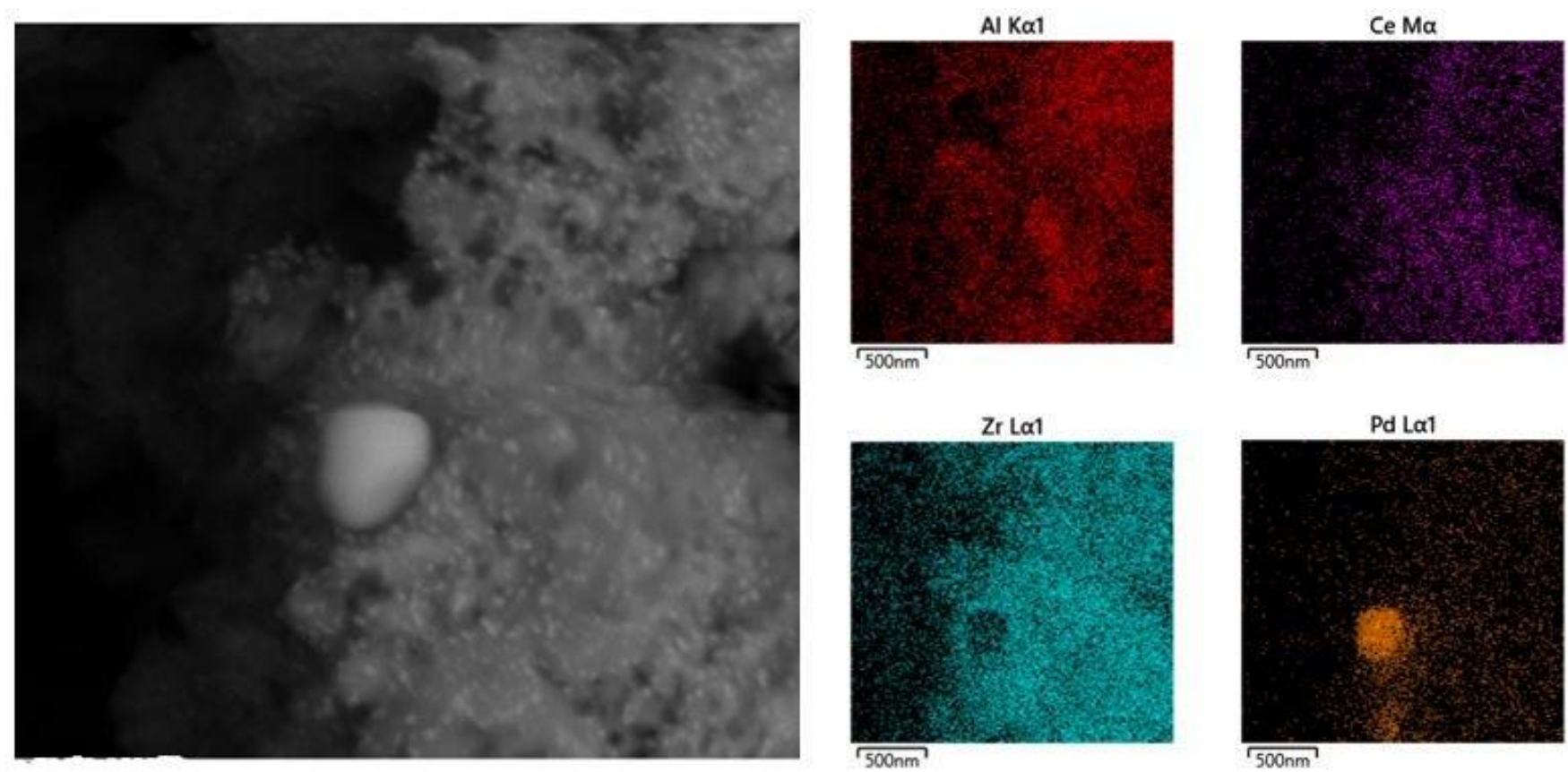

Figure 6. SEM-HR image registered on FUL sample and EDX mapping for $\mathrm{Al}, \mathrm{Ce}, \mathrm{Zr}$ and $\mathrm{Pd}$ recorded on the same area.

Table 1. Minimum, maximum and average value of noble metal particles diameter measured via SEM.

\begin{tabular}{cccc}
\hline & d Min (nm) & d Max (nm) & Average (nm) \\
\hline $25,000 \mathrm{~km}$ & 74 & 115 & 92 \\
\hline $60,000 \mathrm{~km}$ & 102 & 149 & 121 \\
\hline $80,000 \mathrm{~km}$ & 131 & 197 & 150 \\
\hline FUL & 210 & 800 & 398 \\
\hline Lab-aged & 85 & 177 & 130 \\
\hline
\end{tabular}

\subsection{Functional Characterization of Catalysts Samples}

We consider now the functional tests performed on the samples: Figure 7 presents the light off temperatures (T50) for the oxidation of $\mathrm{CO}$ and $\mathrm{HC}$, recorded during the efficiency tests and the results of the oxygen storage capacity (OSC) determination. The light off temperatures increase with ageing for both the reactions, highlighting the effect of catalysts deactivation. The differences of T50 between $\mathrm{CO}$ and $\mathrm{HC}$ are small, less than $10{ }^{\circ} \mathrm{C}$ in almost all the samples tested, except in the case of the FUL sample, which shows a much higher light off temperature for $\mathrm{HC}$ than for $\mathrm{CO}$ abatement.

The oxygen storage capacity decreases significantly upon ageing (Figure $7 \mathrm{~b}$ ). The OSC for the $25,000 \mathrm{~km}$ sample is half of that of the fresh one. Similarly, we observe almost a halving of the OSC between $25,000 \mathrm{~km}$ and $60,000 \mathrm{~km}$. This phenomenon is linked to the redox properties of the oxygen storage components that were found to drastically change with increasing ageing, due to thermal and chemical ageing, as already highlighted by LNT FT-IR measurements. In terms of performances, the Lab-aged samples are quite similar to the $60,000 \mathrm{~km}$ sample, with almost equal light-off temperatures. OSC displays some differences between these samples, with lower values for vehicle-aged samples, probably due to the chemical ageing contribution. In the FUL sample, the oxygen storage capacity is completely compromised. 


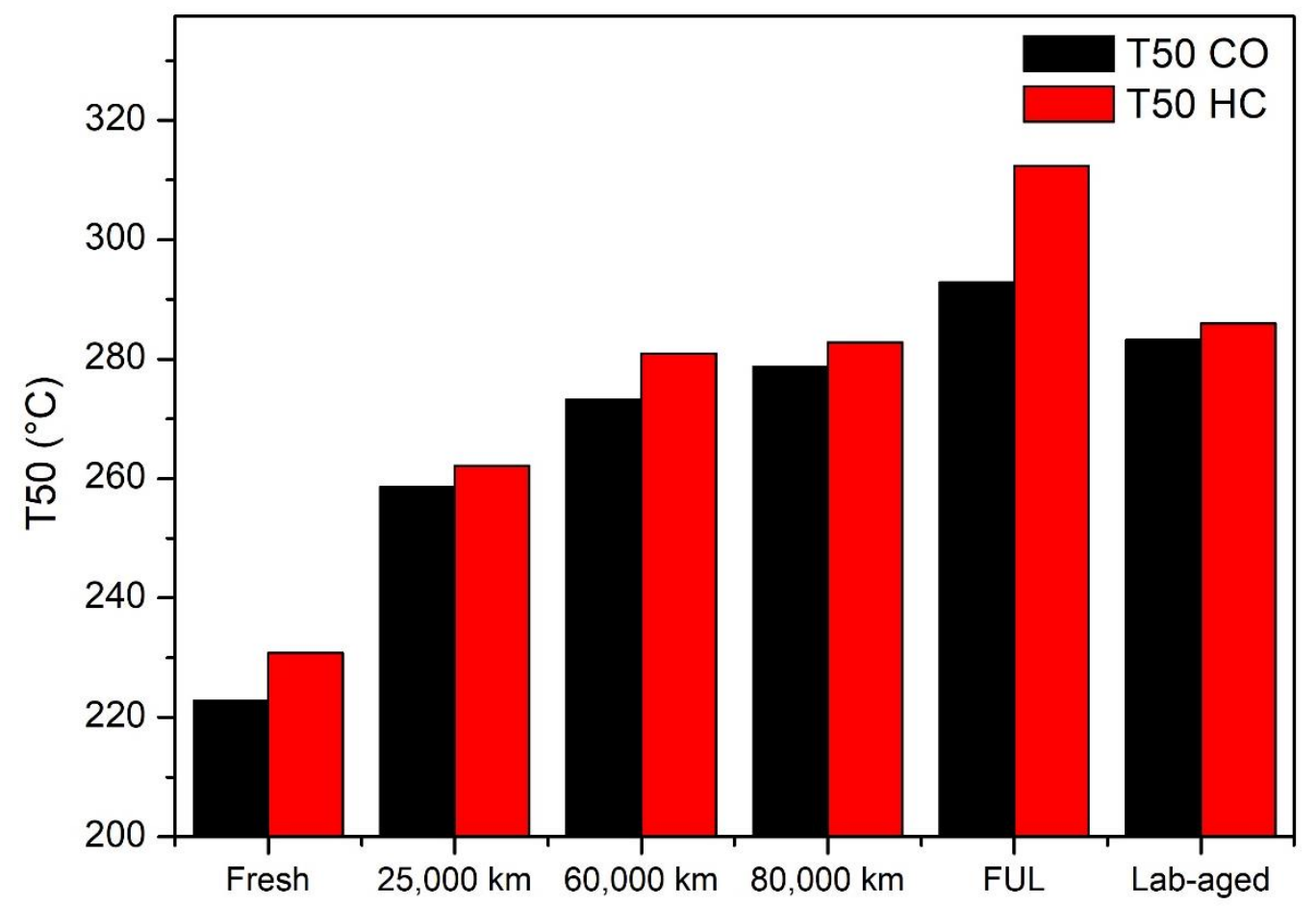

(a)

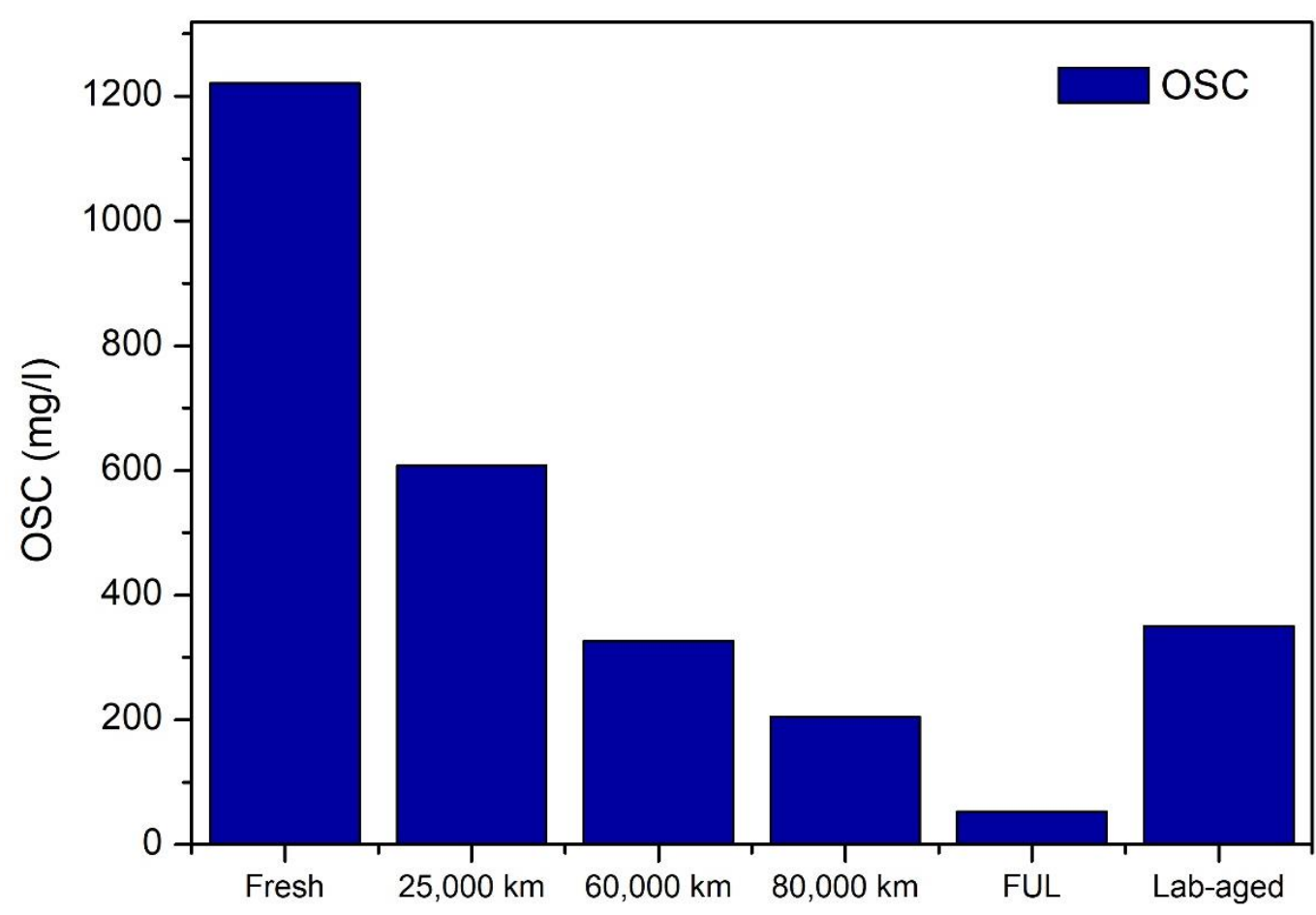

(b)

Figure 7. Light-off temperature (a) and Oxygen storage capacity (b) measured on catalysts samples.

\section{Discussion}

The characterization of all the samples through the applied techniques allows observing the evolution of the catalyst upon ageing, providing a consistent set of qualitative and quantitative data. Sintering of the noble metal particles and Ce-Zr oxides support leads 
to a loss of catalytic functionality. The Pd particles undergo rapid agglomeration, with a rapid loss of dispersion and increase of particles radius. This leads to a loss of active sites, as proven by FT-IR measurements at RT and LNT, with a progressive disappearance of signals associated to the vibrational mode of $\mathrm{CO}$ molecules interacting with noble metal active sites. These physical changes are reflected in an increase of the temperature of light off measured during synthetic gas bench tests. In addition, the FTIR measurement at liquid nitrogen temperature allows to observe the sintering of $\mathrm{Ce}-\mathrm{Zr}$ oxides, but also the contribution of the chemical ageing phenomena, which correlate with a loss of oxygen storage capacity of the materials. This is particularly true for $60,000 \mathrm{~km}$ sample, where it is possible to observe the combination of these two effects. By comparing the on-vehicle and the lab-aged samples, we conclude that the $60,000 \mathrm{~km}$ and laboratory aged samples are very similar in terms of Pd sintering, while the lab aged shows a lower contribution of support ageing, probably due to absence of chemical ageing effects. Also in terms of catalytic performance and catalyst morphology, there are similarities between these two samples. Some differences remain due to the chemical ageing, which was not possible to replicate on the laboratory scale. Another interesting conclusion is that the FUL engine bench aged sample represents a limit case, displaying results very far from both the vehicle-aged and the laboratory-aged samples. This is remarkable considering the fact that the oven ageing methods were originally conceived as a laboratory FUL testing method. On the contrary, they proved to be more representative of an intermediate mileage ageing. We recognize that other factors, not studied in this work, may affect the catalyst performance upon ageing: the evolution of the Rh component of the metal particles, coke formation, and the evolution of the acid-base properties of the support. These additional factors belong to a higher level of detail as compared with the main phenomena studied in this work. Nevertheless, the data we have collected represents a reference framework for the development of improved laboratory ageing protocols, meeting the demand of an increased catalyst lifetime.

\section{Materials and Methods}

\subsection{Catalysts}

The chosen catalyst technology, already used in our previous work [29], is a commercial formulation, based on double metal Pd/Rh technology (55:5), with a total PGM loading of $60 \mathrm{~g} / \mathrm{ft}^{3}$ (1 wt.\%), supported on a porous substrate mainly composed by Ce, $\mathrm{Zr}$ and Al oxides, with dopants (Ba, La). The component, a cylindrical ceramic monolith of $0.55 \mathrm{~L}$ $\left(4.2^{\prime \prime} \times 2.5^{\prime \prime}\right)$ characterized by a high density of channels (900 cpsi) with hexagonal shape, was core drilled to obtain two cylindrical samples of $1.5^{\prime \prime}$ of diameter and $2.5^{\prime \prime}$ length, one for the morphological characterization, one for the functional testing.

The reference real samples came from two vehicles equipped with $1.2 \mathrm{~L}, 69 \mathrm{hp}$ (Horse Power) gasoline engines compliant with the E6 b emission standard. The ageing mileage were 25,000,60,000 and 80,000 km. A sample came from an engine bench ageing cycle: an engine-dynamometer set-up (the same engine of the vehicle) used to perform an accelerated ageing protocol to simulate a catalyst aged at full useful life of the vehicle $160,000 \mathrm{~km}$, in severe driving condition (denoted as Full Useful Life (FUL) in the paper).

For the laboratory deactivation protocol, a sample was core-drilled from a fresh monolith and aged using a tubular oven equipped with a gas line and a peristaltic pump, in order to flux a mixture of the air and water vapor in the sample during the ageing operation. The temperature and the time of ageing: were $1100^{\circ} \mathrm{C}$ for $7 \mathrm{~h}$, a common industrial standard to simulate an end-of-life condition in hydrothermal oxidizing environment. The ageing was performed in pure air flux $(4 \mathrm{~L} / \mathrm{min}$ ), with $10 \%$ water (sample denoted as Lab-aged in the paper). All the cored samples extracted from the fresh monolith were conditioned, before undergoing testing, in a static oven at $650{ }^{\circ} \mathrm{C}$ for $2 \mathrm{~h}$ to eliminate any possible residue of production intermediates. 


\subsection{Noble Metal Characterization Techniques}

The washcoat was extracted from the first batch of cored sample, in order to remove the contribution of the ceramic support, unnecessary to ageing phenomena characterization. This extraction was performed using a method developed by some of us and described in a previous work [38], based on the detachment of the material by thermal shock, performed soaking the sample first in liquid nitrogen, then in water, blowing with gaseous $\mathrm{N}_{2}$ to remove water residues from channels, to avoid substrate detachment due to ice formation. The washcoat extracted was dried obtaining a fine powder.

Washcoat powders were compressed in self-supporting discs $\left(\sim 20 \mathrm{mg} \mathrm{cm}^{-2}\right)$ and placed in IR cells suitable for high-temperature treatments and FT-IR analysis at room and liquid-nitrogen temperature (RT and LNT, respectively). Measurements performed at LNT allow to characterize the metal phase and the oxidic support as well. Before the IR measurements, the samples were pretreated, following two different protocols. The first one was a treatment in vacuum at $400{ }^{\circ} \mathrm{C}$ for $30 \mathrm{~min}$. The second one was an activation protocol through oxidation and subsequent reduction of the material. The oxidation step was performed in a static system using dry $\mathrm{O}_{2}$ on the sample at $400{ }^{\circ} \mathrm{C}$. Oxygen was used twice, at about $40 \mathrm{mbar}$ for $15 \mathrm{~min}$ per each step: the first treatment was useful to remove reduced species, possibly adsorbed on the surface; the second treatment was performed to ensure the oxidation. The reduction step was performed in the same way as the previous one, but the treatment was carried out in $\mathrm{H}_{2}$. Hydrogen was used twice at $40 \mathrm{mbar}$ for 15 min per each step. The double treatment was intended to ensure the reduction. Finally, the sample was outgassed and cooled to RT in vacuum. Absorption/transmission IR spectra were run on a Perkin-Elmer FTIR System 2000 spectrophotometer (Perkin-Elmer, Waltham, MA, USA) equipped with a Hg-Cd-Te cryo-detector, working in the range of wavenumbers $7200-580 \mathrm{~cm}^{-1}$ at a resolution of $2 \mathrm{~cm}^{-1}$, with 60 scans. IR absorbance spectra were acquired at RT and LNT on the pretreated samples at increasing CO pressure up to 20 mbar.

The distribution and the dispersion of noble metal particles in washcoat samples were analyzed via pulse $\mathrm{CO}$ chemisorption. These analyses were performed by using a Thermo Scientific 1100 TPDRO (Thermo Fisher Scientific, Waltham, MA, USA) equipped with a thermal conductivity detector (TCD). The catalyst powder $(200 \mathrm{mg})$ was placed in a quartz reactor, between two quartz wool layers. A reducing pre-treatment was carried out in a $20 \mathrm{~mL} \mathrm{~min}^{-1}$ flow of $5 \% \mathrm{H}_{2}$ in $\mathrm{Ar}$, by heating the sample up to $300{ }^{\circ} \mathrm{C}$ with a $10^{\circ} \mathrm{C} / \mathrm{min}$ ramp and maintaining this temperature for $120 \mathrm{~min}$. CO chemisorption was performed at room temperature, using He as carrier gas $\left(20 \mathrm{~mL} \mathrm{~min}^{-1}\right.$ flow) and sending pulses of $800 \mu \mathrm{L}$ of a mixture of $10 \% \mathrm{CO}$ in He until saturation [26]. TCD detects differences in the thermal conductivity between the gas entering and leaving the reactor. Therefore, to detect changes in $\mathrm{CO}$ concentration, He must be used as a carrier gas, since it has a very different thermal conductivity. During the test, He flows continuously and every 10-15 min a CO injection is made into the He flow. After a few minutes the detector reveals a signal proportional to the $\mathrm{CO}$ output concentration (the presence of this gas lowers the average thermal conductivity of the mixture). Each pulse is therefore recorded as a TCD peak whose area is proportional to the non-adsorbed CO. If chemisorption occurs, the area of the peaks is initially smaller, and then it gradually increases until saturation of the surface. The amount of chemisorbed $\mathrm{CO}$ on the sample can be calculated from the integration of the peaks. From this datum, it is possible to estimate the metal dispersion [28].

In order to observe noble metals particles and follow their evolution during the ageing, all the samples were observed using a field emission scanning electron microscope: a FESEM TESCAN S9000G (TESCAN ORSAY HOLDING, Brno, Czechia) equipped with a Schottky type FEG source. The detectors were an In-Beam Secondary Electron (SE) detector with a resolution of $0.7 \mathrm{~nm}$ at $15 \mathrm{keV}$, and a back scattered electron detector (BSD). Elemental analysis was measured with an EDX detector Ultima Max OXFORD (Oxford Instruments, Abingdon, UK) with AZTEC software. 


\subsection{Catalyst Functional Characterization}

The second set of cored samples was prepared and used to evaluate the catalytic performances, performed on the full component (support + washcoat). The samples were tested on a synthetic gas bench specifically designed for functional testing of automotive catalysts (Figure 8). A synthetic gas mixture $\left(0.7 \% \mathrm{O}_{2}, 15 \% \mathrm{CO}, 10 \% \mathrm{CO}_{2}, 1875 \mathrm{ppm} \mathrm{HC}\right.$, $600 \mathrm{ppm} \mathrm{NO}$ and $\left.1 \% \mathrm{H}_{2} \mathrm{O}\right)$, based on a carrier flow of $\mathrm{N}_{2}\left(\mathrm{SV}=50 \mathrm{k} \mathrm{h}^{-1}\right)$ was heated to the desired temperature and fed to the heated catalyst. The gas mixture was analyzed sampling both before and after the catalyst, in order to evaluate the flux composition and its variation during the reactions. This analysis was performed using a FT-IR Multi-gas Analyzer MKS 2030 HS for the simultaneous analysis of all chemical species. The gas mixture composition was studied to replicate typical gasoline engine emissions, using a $\mathrm{HC}$ mixture composed by of short-chain hydrocarbons (ethylene, propylene and methane $3,5 / 1 / 1)$. This system is not sufficiently fast to replicate the fluctuation between lean (oxidant) and rich (reducing) conditions, so it works near the stoichiometric condition $(\lambda=1)$ in a slightly lean environment. Two types of test were performed: first, the conversion efficiency of $\mathrm{CO}$ and $\mathrm{HC}$ (due to lean environment chosen) was measured flowing the feed gas mixture over the sample upon a temperature increase (ramp rate of $30^{\circ} \mathrm{C} / \mathrm{min}$ from ambient temperature to $500{ }^{\circ} \mathrm{C}$ ). From this result, the light-off temperature (T50) of the catalyst was obtained, defined as the temperature in which the catalyst reaches $50 \% \mathrm{CO}$ or HC conversion efficiency [39]. The second test was focused on the evaluation of the oxygen storage capacity (OSC) of the component, whose reduction upon ageing is extremely detrimental to catalyst functionality. This measurement was performed at constant temperature $\left(400^{\circ} \mathrm{C}\right)$, saturating the sample with $\mathrm{O}_{2}$ and subsequently measuring the $\mathrm{CO}$ consumption upon dosage of a CO-rich feed (7000-8000 ppm). All the tests were performed three times, in order to verify the reproducibility of the results.

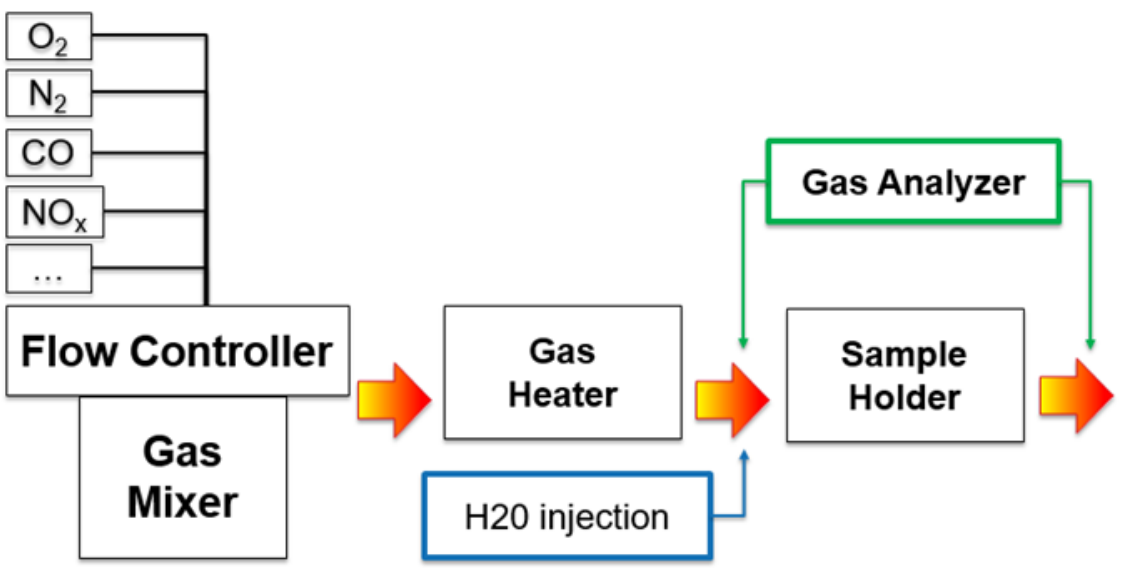

Figure 8. Schematic layout of the Synthetic Gas Bench (SGB) used for the catalyst testing.

Author Contributions: M.G., M.S. and G.R. designed the project, M.G. developed and performed washcoat extraction methodology and SGB analysis; M.C.V. and M.G. performed FE-SEM analysis; E.S. and S.B. designed and performed CO chemisorption and Pd particles dispersion calculation; P.T. and S.M. performed FT-IR analysis; M.G. wrote the manuscript in consultation with M.S. and G.R. All authors have read and agreed to the published version of the manuscript.

Funding: This research received no external funding.

Conflicts of Interest: The authors declare no conflict of interest.

\section{References}

1. Heck, R.M.; Farrauto, R.J.; Gulati, S.T. Catalytic Air Pollution Control: Commercial Technology; John Wiley \& Sons: Hoboken, NJ, USA, 2016.

2. Wang, J.; Chen, H.; Hu, Z.; Yao, M.; Li, Y. A Review on the Pd-Based Three-Way Catalyst. Catal. Rev. 2015, 57, 79-144. [CrossRef] 
3. Machida, M.; Fujiwara, A.; Yoshida, H.; Ohyama, J.; Asakura, H.; Hosokawa, S.; Tanaka, T.; Haneda, M.; Tomita, A.; Miki, T.; et al. Deactivation Mechanism of Pd/CeO2-ZrO2 Three-Way Catalysts Analyzed by Chassis-Dynamometer Tests and in Situ Diffuse Reflectance Spectroscopy. ACS Catal. 2019, 9, 6415-6424. [CrossRef]

4. González-Velasco, J.R.; Botas, J.A.; Ferret, R.; González-Marcos, M.P.; Marc, J.-L.; Gutiérrez-Ortiz, M.A. Thermal aging of $\mathrm{Pd} / \mathrm{Pt} / \mathrm{Rh}$ automotive catalysts under a cycled oxidizing-reducing environment. Catal. Today 2000, 59, 395-402. [CrossRef]

5. Winkler, A.; Ferri, D.; Hauert, R. Influence of aging effects on the conversion efficiency of automotive exhaust gas catalysts. Catal. Today 2010, 155, 140-146. [CrossRef]

6. Vedyagin, A.A.; Volodin, A.M.; Kenzhin, R.M.; Stoyanovskii, V.O.; Rogov, V.A.; Medvedev, D.A.; Mishakov, I.V. Characterization and study on the thermal aging behavior of palladium-alumina catalysts. J. Therm. Anal. Calorim. 2017, 130, 1865-1874. [CrossRef]

7. Neyestanaki, A.K.; Klingstedt, F.; Salmi, T.; Murzin, D.Y. Deactivation of postcombustion catalysts, a review. Fuel 2004, 83, 395-408. [CrossRef]

8. More, K.L.; Kenik, E.A.; Coffey, D.W.; Geer, T.S.; LaBarge, W.J.; Beckmeyer, R.F.; Theis, J. Thermally-Induced Microstructural Changes in a Three-Way Automotive Catalyst. SAE Tech. Paper Ser. 1997, 1255-1263. [CrossRef]

9. Zheng, T.; He, J.; Zhao, Y.; Xia, W.; He, J. Precious metal-support interaction in automotive exhaust catalysts. J. Rare Earths 2014, 32, 97-107. [CrossRef]

10. Graham, G.; Jen, H.-W.; Chun, W.; McCabe, R. High-Temperature-Aging-Induced Encapsulation of Metal Particles by Support Materials: Comparative Results for Pt, Pd, and Rh on Cerium-Zirconium Mixed Oxides. J. Catal. 1999, 182, 228-233. [CrossRef]

11. Farrauto, R.; Hobson, M.; Kennelly, T.; Waterman, E. Catalytic chemistry of supported palladium for combustion of methane. Appl. Catal. A Gen. 1992, 81, 227-237. [CrossRef]

12. Theis, J.R.; McCabe, R.W. The effects of high temperature lean exposure on the subsequent HC conversion of automotive catalysts. Catal. Today 2012, 184, 262-270. [CrossRef]

13. Schoenhaber, J.; Kuehn, N.; Bradler, B.; Richter, J.M.; Bauer, S.; Lenzen, B.; Beidl, C. Impact of european real-driving-emissions legislation on exhaust gas aftertreatment systems of turbocharged direct injected gasoline vehicles. SAE Tech. Paper. 2017, 1, 0924.

14. Kang, S.B.; Han, S.J.; Nam, S.B.; Nam, I.-S.; Cho, B.K.; Kim, C.H.; Oh, S.H. Effect of Aging Atmosphere on Thermal Sintering of Modern Commercial TWCs. Top. Catal. 2013, 56, 298-305. [CrossRef]

15. Sheng, S.; Lai, Y.; Hao, C.; Hou, P.; Lv, T.; Ge, Y. Review of rapid ageing testing methods of three-way catalyst for gasoline engine. Int. J. Veh. Perform. 2020, 6, 277-293.

16. Shelef, M.; Otto, K.; Otto, N. Poisoning of Automotive Catalysts. Adv. Catal. 1979, 27, 311-365. [CrossRef]

17. Kang, S.B.; Kwon, H.J.; Nam, I.-S.; Song, Y.I.; Oh, S.H. Activity Function for Describing Alteration of Three-Way Catalyst Performance over Palladium-Only Three-Way Catalysts by Catalyst Mileage. Ind. Eng. Chem. Res. 2011, 50, 5499-5509. [CrossRef]

18. Stenbom, B.; Smedler, G.; Nilsson, P.H.; Lundgren, S.; Wirmark, G. Thermal Deactivation of a Three-Way Catalyst: Changes of Structural and Performance Properties. SAE Tech. Paper Ser. 1990. [CrossRef]

19. Hietikko, M.; Lassi, U.; Kallinen, K.; Savimäki, A.; Härkönen, M.; Pursiainen, J.; Laitinen, R.; Keiski, R. Effect of the ageing atmosphere on catalytic activity and textural properties of Pd/Rh exhaust gas catalysts studied by XRD. Appl. Catal. A Gen. 2004, 277, 107-117. [CrossRef]

20. Lassi, U.; Polvinen, R.; Suhonen, S.; Kallinen, K.; Savimäki, A.; Härkönen, M.; Valden, M.; Keiski, R. Effect of ageing atmosphere on the deactivation of $\mathrm{Pd} / \mathrm{Rh}$ automotive exhaust gas catalysts: Catalytic activity and XPS studies. Appl. Catal. A Gen. 2004, 263, 241-248. [CrossRef]

21. $\mathrm{Wu}, \mathrm{X} . ; \mathrm{Xu}, \mathrm{L} . ; \mathrm{Weng}, \mathrm{D}$. The thermal stability and catalytic performance of Ce-Zr promoted Rh-Pd/ $\gamma$-Al2O3 automotive catalysts. Appl. Surf. Sci. 2004, 221, 375-383. [CrossRef]

22. Hadjivanov, K.I.; Vayssilov, G.N. Characterization of Oxide Surfaces and Zeolites by Carbon Monoxide as an IR Probe. Mol. Chem. 2003, 34, 307-511. [CrossRef]

23. Terekhina, O.; Roduner, E. FTIR Spectroscopic Investigation of Zeolite-Supported Pd-Ag Bimetallic Clusters. J. Phys. Chem. C 2012, 116, 6973-6979. [CrossRef]

24. Fernández-García, M.; Martínez-Arias, A.; Iglesias-Juez, A.; Hungría, A.B.; Anderson, J.A.; Conesa, J.C.; Soria, J. New $\mathrm{Pd} / \mathrm{CexZr1}-\mathrm{xO} 2 / \mathrm{Al} 2 \mathrm{O} 3$ three-way catalysts prepared by microemulsion: Part 1 . Characterization and catalytic behavior for CO oxidation. Appl. Catal. B Environ. 2001, 31, 39-50. [CrossRef]

25. Groppo, E.; Bertarione, S.; Rotunno, F.; Agostini, G.; Scarano, D.; Pellegrini, R.; Leofanti, G.; Zecchina, A.; Lamberti, C. Role of the Support in Determining the Vibrational Properties of Carbonyls Formed on Pd Supported on $\mathrm{SiO} 2-\mathrm{Al} 2 \mathrm{O} 3, \mathrm{Al} 2 \mathrm{O} 3$, and MgO. J. Phys. Chem. C 2007, 111, 7021-7028. [CrossRef]

26. Bensalem, A.; Muller, J.-C.; Tessier, D.; Bozon-Verduraz, F. Spectroscopic study of CO adsorption on palladium-ceria catalysts. J. Chem. Soc. Faraday Trans. 1996, 92, 3233-3237. [CrossRef]

27. Lynn, M.A.; Bruce, E.B. An analysis of the bonding in some 'nonclassical'd0 and d10 metal carbonyl complexes. Inorgan. Chim. Acta 1995, 229, 437-443. [CrossRef]

28. Bergeret, G.; Gallezot, P. Particle size and dispersion measurements. In Handbook of Heterogeneous Catalysis; Wiley Online Library: Hoboken, NJ, USA, 2008; pp. 738-765.

29. Giuliano, M.; Ricchiardi, G.; Valsania, M.C.; Parussa, F.; Nicol, G.; Sgroi, M. Characterization of Vehicle and Laboratory Aged Commercial Three Way Catalyst: A Morphological and Functional Correlation between Real and Simulated Ageing. Int. J. Automot. Technol. 2021, 22, 131-139. [CrossRef] 
30. Vedyagin, A.A.; Volodin, A.M.; Stoyanovskii, V.O.; Kenzhin, R.M.; Slavinskaya, E.M.; Mishakov, I.V.; Plyusnin, P.E.; Shubin, Y.V. Stabilization of active sites in alloyed Pd-Rh catalysts on $\gamma$-Al2O3 support. Catal. Today 2014, 238, 80-86. [CrossRef]

31. Surnev, S.; Sock, M.; Ramsey, M.G.; Netzer, F.P.; Wiklund, M.; Borg, M.; Andersen, J.N. CO adsorption on Pd(111): A highresolution core level photoemission and electron energy loss spectroscopy study. Surf. Sci. 2000, 470, 171-185. [CrossRef]

32. Xu, X.; Goodman, D.W. An infrared and kinetic study of carbon monoxide oxidation on model silica-supported palladium catalysts from 10-9 to 15 Torr. J. Phys. Chem. 1993, 97, 7711-7718. [CrossRef]

33. Hollins, P. The influence of surface defects on the infrared spectra of adsorbed species. Surf. Sci. Rep. 1992, 16, 51-94. [CrossRef]

34. Gong, J.; Wang, D.; Li, J.; Kamasamudram, K.; Currier, N.; Yezerets, A. An experimental and kinetic modeling study of aging impact on surface and subsurface oxygen storage in three-way catalysts. Catal. Today 2019, 320, 51-60. [CrossRef]

35. Christou, S.; Alvarez-Galvan, M.C.; Fierro, J.; Efstathiou, A. Suppression of the oxygen storage and release kinetics in Ce0.5Zr0.5O2 induced by P, Ca and Zn chemical poisoning. Appl. Catal. B Environ. 2011, 106, 103-113. [CrossRef]

36. Borodziński, A.; Magdalena, B. Relation between crystallite size and dispersion on supported metal catalysts. Langmuir 1997, 13, 5613-5620. [CrossRef]

37. Beck, A.; Horváth, A.; Szücs, A.; Schay, Z.; Horváth, Z.; Zsoldos, Z.; Dékány, I.; Guczi, L. Pd nanoparticles prepared by “controlled colloidal synthesis" in solid/liquid interfacial layer on silica. I. Particle size regulation by reduction time. Catal. Lett. 2000, 65, 33-42. [CrossRef]

38. Giuliano, M.; Ricchiardi, G.; Damin, A.; Sgroi, M.; Nicol, G.; Parussa, F. Thermal Ageing Effects in a Commercial Three-Way Catalyst: Physical Characterization of Washcoat and Active Metal Evolution. Int. J. Automot. Technol. 2020, 21, 329-337. [CrossRef]

39. Fernandes, D.M.; Neto, A.A.; Cardoso, M.J.B.; Zotin, F.M.Z. Commercial automotive catalysts: Chemical, structural and catalytic evaluation, before and after aging. Catal. Today 2008, 133, 574-581. [CrossRef] 